\title{
Australian general practitioners' attitudes and knowledge of sentinel lymph node biopsy in melanoma management
}

Caroline G Watts, Andrea L Smith, Sam Robinson, Chiao-Han Chang, Chris Goumas, Helen Schmid, John W Kelly, Angela M Hong, Richard A Scolyer, Georgina V Long, Andrew J Spillane, Michael Henderson, David E Gyorki, Victoria J Mar, Rachael L Morton, Robyn PM Saw, Alex H Varey, Graham J Mann, John F Thompson, Anne E Cust

\section{Background and objective}

In Australia, the uptake of the sentinel lymph node biopsy (SLNB) appears low despite clinical practice guideline recommendations. The aim of this study was to describe the knowledge and attitudes of general practitioners (GPS) to SLNB.

\section{Methods}

GPs were recruited at an annual conference and a skin cancer skills workshop, and using GP professional communications. A mixed methods approach comprised a cross-sectional questionnaire and, for a subset of participants, semi-structured interviews.

\section{Results}

Overall, 231 GPs completed the questionnaire, of whom 23 were interviewed. One-third (32\%) described themselves as quite or very familiar with the guidelines, and two-thirds (68\%) thought that SLNB had an important role in the management of patients with melanoma. Of GPs who would discuss SLNB with eligible patients, $<40 \%$ correctly identified that SLNB is recommended for patients with an invasive melanoma $>1 \mathrm{~mm}$ thick.

\section{Conclusions}

GPs were generally supportive of SLNB. Familiarity with the guidelines was low, particularly regarding which patients should be considered for SLNB.
IN AUSTRALIA, general practitioners (GPs) are the first point of contact for the majority of patients who develop melanoma. A GP is therefore the person who will most often make a preliminary diagnosis, take the first biopsy and, following pathological confirmation of melanoma, decide on the need for referral for specialist care. Some GPs with a special interest in skin cancer also perform wide excisions and flap repairs. ${ }^{1}$ The NSW Melanoma Patterns of Care study, ${ }^{2}$ a populationbased survey of in situ and invasive melanoma management in NSW in 2006-07, found the initial melanoma diagnosis was managed in general practice for $36 \%$ of patients, in skin cancer clinics (usually staffed by GPs) for $17 \%$, in dermatology practices for $26 \%$, by surgeons for $13 \%$ and by others for $<1 \%$. Approximately half of patients initially managed by a GP are then referred to a specialist or multidisciplinary centre. ${ }^{2}$

Melanoma prognosis is closely related to whether a melanoma has spread to regional or distant parts of the body. Sentinel lymph node biopsy (SLNB) was introduced in $1992^{3}$ and is performed after lymphatic mapping to provide information about the presence of metastatic melanoma in the regional lymph node basin. SLNB is important for staging and prognostic assessment, and to guide patient management including appropriateness for systemic adjuvant therapies and clinical trials. ${ }^{4}$ To optimise the accuracy of the test, SLNB should be performed in a centre with expertise in the procedure, including specialist nuclear medicine, surgery and pathology. ${ }^{5}$

Current Australian guidelines recommend that SLNB be considered for all patients with melanoma $>1 \mathrm{~mm}$ in thickness and for patients with melanoma $>0.8 \mathrm{~mm}$ in thickness with other high-risk pathological features. ${ }^{5}$ SLNB should be performed at the time of the primary tumour wide excision. ${ }^{5}$ Historically, adherence to SLNB guidelines has not been optimal, allowing for patients who may refuse or not be suitable for SLNB. Data from the NSW Melanoma Patterns of Care study indicated that SLNB was undertaken for $45 \%$ of patients diagnosed with a melanoma $>0.8 \mathrm{~mm}$ Breslow thickness who 
were potentially eligible for SLNB. ${ }^{6}$ The Multicenter Selective Lymphadenectomy Trial (MSLT-I) showed improved disease-free survival but not overall melanoma-specific survival following SLNB. ${ }^{7}$ There have been conflicting interpretations of the results from MSLT-I and other studies examining the benefits of SLNB in relation to the therapeutic value $^{8,9}$ and survival benefit of SLNB. ${ }^{10,11}$ Potential reasons for low uptake of SLNB include confusion about the evidence, lack of awareness of the guidelines, and individual preferences of GPs and patients. The aim of this study was to examine the knowledge and attitudes of GPs regarding the role of SLNB in the management of patients with invasive primary melanomas, to assist development of and adherence to guidelines.

\section{Methods}

Mixed methods study design Quantitative and qualitative data were collected in the form of questionnaires and interviews. Informed consent was obtained.

\section{Sampling and recruitment}

Recruitment of GPs was conducted at two Australian GP meetings: the Royal Australian College of General Practitioners (RACGP) annual conference in Queensland in October 2018, and a GP skin cancer-focused continuing medical education workshop in Sydney in December 2018; participants were also recruited through other GP professional communications. GPs were eligible to participate in the study if they had practised in Australia in the previous year. Overall, 231 GPs completed a questionnaire. Of these, 23 also completed an interview and were reimbursed $\$ 100$ for their time.

\section{Questionnaire and interview guide development}

A cross-sectional questionnaire and semi-structured interview guide (Supplementary file; available online only) were developed from a literature review and discussion with a multidisciplinary team of melanoma clinicians and researchers. The questionnaire was completed on paper or electronically and consisted of 24 items covering demographic characteristics, knowledge of melanoma guidelines, clinical management of melanoma, referral patterns, and attitudes to SLNB and shared care. The questionnaire data were managed using REDCap. ${ }^{12}$

\section{Questionnaire data analysis}

Postcodes were classified using the Accessibility Remoteness Index of Australia (ARIA) ${ }^{13}$ and Socio-Economic Indexes for Areas (SEIFA) ${ }^{14}$ classifications. Univariable and multivariable prevalence ratios and $95 \%$ confidence intervals (CIs) were calculated using Poisson regression models to examine factors associated with familiarity with Australian melanoma clinical practice guidelines and knowledge about and attitudes towards SLNB.

Factors assessed were age, sex, type of practice, years of practice, number of invasive melanomas diagnosed in a year, ARIA, SEIFA and exposure to articles or talks about SLNB. Analyses were conducted using SAS version 9.4. The STROBE guidelines were followed to report the findings. ${ }^{15}$

\section{Qualitative data analysis}

Interviews (conducted by SR and ALS) lasted 16-39 minutes (mean length: 24 minutes) and were audio-recorded and professionally transcribed. The de-identified transcripts were read by two members of the research team (SR and ALS). Data were compared within and across interviews to identify commonalities, differences and patterns in the data and to develop a coding framework and thematic map. ${ }^{16}$ The codes and themes were discussed with the research team and refined until consensus was reached. Coding was conducted using NVivo12 (QSR International, Australia) software.

\section{Results}

\section{Participant characteristics}

Characteristics of GPs who participated in the study are shown in Table 1 . Of the GPs who completed the questionnaire, $46 \%$ worked in group medical practices, $32 \%$ in independent practice, $14 \%$ in skin cancer clinics and $8 \%$ in other types of practice. The majority of GPs (83\%) diagnosed fewer than six patients presenting with melanoma per year.

\section{Familiarity with Australian clinical practice guidelines for SLNB}

\section{Questionnaire results}

Self-reported familiarity with the guidelines for melanoma management varied: $30 \%$ were unfamiliar or somewhat unfamiliar; $38 \%$ were a little familiar; and $32 \%$ were quite familiar or very familiar. Overall, $31 \%$ of GPs reported they had checked the recent update of the melanoma guidelines. In univariable analyses, GPs were more likely to claim familiarity with the guidelines if they saw $\geq 6$ patients/ year with melanoma when compared with $\leq 5$ patients/year (prevalence ratio 2.84; 95\% CI: $1.77,4.55)$, worked in a skin cancer clinic when compared with medical centre (prevalence ratio 2.90; 95\% CI: $1.60,5.24$ ) or had been exposed to articles or talks about SLNB (prevalence ratio 4.19; 95\% CI: 2.15, 8.18). In multivariable analysis, exposure to articles or talks about SLNB was the only factor that remained statistically significantly associated with familiarity of the guidelines (prevalence ratio 3.55; 95\% CI: 1.71, 7.39).

Of the 164 GPs (72\%) who reported that they would discuss SLNB and would refer eligible patients for discussion of the risks and benefits of SLNB with a surgeon (Table 2), 39\% indicated they would tell a patient that SLNB would be appropriate if a melanoma's Breslow thickness was >1.0-2.0 mm; responses for different Breslow thickness criteria are shown in Table 3. Three-quarters of GPs indicated they would arrange other tests if their patient was eligible for SLNB. The most commonly ordered tests were computed tomography (CT) of the chest, abdomen and pelvis (36\%); whole-body positron emission tomography (PET)/CT (28\%); and regional node ultrasonography (18\%; Table 3), despite the Australian guidelines recommending no investigations prior to SLNB. 


\begin{tabular}{|c|c|c|}
\hline Characteristic & Questionnaire n (\%) & Interview n (\%) \\
\hline \multicolumn{3}{|l|}{ Sex } \\
\hline Male & $132(57)$ & $12(52)$ \\
\hline Female & $99(43)$ & $11(48)$ \\
\hline \multicolumn{3}{|l|}{ Age group (years) } \\
\hline$<30$ & $17(7)$ & $2(9)$ \\
\hline $30-49$ & $131(57)$ & $12(52)$ \\
\hline $50-69$ & $76(33)$ & $9(39)$ \\
\hline$\geq 70$ & $7(3)$ & $0(0)$ \\
\hline \multicolumn{3}{|l|}{ Practice type } \\
\hline Independent practice* & $74(32)$ & $10(43)$ \\
\hline Group medical practice & $107(46)$ & $8(35)$ \\
\hline Skin cancer clinic & $32(14)$ & $4(17)$ \\
\hline Other & $18(8)$ & $1(4)$ \\
\hline \multicolumn{3}{|c|}{ Number of years practising as a GP } \\
\hline$<5$ & $84(37)$ & $9(39)$ \\
\hline $6-10$ & $45(20)$ & $5(22)$ \\
\hline $11-20$ & $32(14)$ & $2(9)$ \\
\hline$>20$ & $69(30)$ & $7(30)$ \\
\hline Missing data & 1 & \\
\hline \multicolumn{3}{|c|}{ Number of patients diagnosed with invasive melanoma in the previous year } \\
\hline 0 & $31(13)$ & $3(13)$ \\
\hline 1 & $69(30)$ & $5(22)$ \\
\hline $2-5$ & $92(40)$ & $10(43)$ \\
\hline $6-10$ & $25(11)$ & $5(22)$ \\
\hline$>10$ & $13(6)$ & $0(0)$ \\
\hline Missing data & 1 & \\
\hline
\end{tabular}

\begin{tabular}{lrrr}
\hline Practice by ARIA index & \\
\hline Highly accessible & $162(72)$ & $14(61)$ \\
\hline Accessible & $36(16)$ & $7(30)$ \\
\hline Less accessible & $26(12)$ & 2 (9) \\
\hline Missing data & 7 &
\end{tabular}

Practice by SEIFA index ${ }^{14}$

\begin{tabular}{lrr}
\hline Q1 (most disadvantaged) & $34(15)$ & $4(17)$ \\
\hline Q2 & $56(25)$ & $5(22)$ \\
\hline Q3 & $71(32)$ & $7(30)$ \\
\hline Q4 (least disadvantaged) & $63(28)$ & $7(30)$ \\
\hline Missing data & 7 &
\end{tabular}

*Solo practice

ARIA, Accessibility/Remoteness Index of Australia; GP, general practitioner; SEIFA, Socio-Economic Indexes for Areas

\section{Attitudes and views about SLNB for melanoma management}

\section{Questionnaire results}

Overall, $68 \%$ of GPs believed that SLNB had an important role in the management of patients with melanoma, $30 \%$ were unsure and $2 \%$ disagreed (Table 2). Most GPs (60\%) had been exposed to articles or talks about SLNB in the past three years (Table 2); in a multivariable analysis, this was associated with less uncertainty about the importance of SLNB (prevalence ratio 0.58; 95\% CI: $0.35,0.96$ ) and less hesitation in discussing SLNB with patients (prevalence ratio OR 0.58; 95\% CI: 0.34, 0.99).

Of the 164 GPs (72\%) who said that they would discuss SLNB with their patients, $71 \%$ indicated a role of SLNB for staging and prognostic information, $67 \%$ thought SLNB was useful to assess suitability for adjuvant systemic therapies for patients who were node-positive, $62 \%$ considered that the results would influence patient management, $40 \%$ considered there was a likely survival benefit for performing SLNB and 30\% indicated that SLNB assisted selection of patients for completion of lymphadenectomy (Table 3). The factors most influential in their decision to discuss or recommend SLNB were Breslow thickness (92\%), lymphovascular invasion (66\%), presence of palpable lymph nodes (67\%) and the likelihood that the result would influence patient management (54\%; Table 3).

Table 3 also presents GPs' preferences for referral of patients for whom SLNB would be applicable for definitive management, and preferences for the degree of involvement in patient follow-up care following a positive or negative SLNB result.

Of the 64 GPs (28\%) who indicated they would not usually discuss SLNB with or recommend SLNB for a patient with newly diagnosed melanoma eligible for SLNB, 47\% said they did not know enough about SLNB. Under the option 'Other, please specify', 29\% reported that recommendations were the role of whoever was caring for the patient's melanoma. Other reasons (not shown) included unconfirmed survival benefit (14\%), access to facilities (9\%) and lack of additional prognostic information (8\%). 


\section{Interview results}

The questionnaire data indicated a substantial proportion of participants were unable to correctly identify the Breslow thickness at which patients should be referred for discussion of SLNB. The interview data (Table 4) provided a possible explanation for this knowledge gap by revealing that many of the GPs did not perceive the SLNB guidelines to be relevant to their practice and many had a limited working knowledge of these guidelines. They frequently expressed the belief that decisions related to SLNB were the responsibility of the specialist and not the GP. The interview data also indicated considerable variation in GPs' knowledge of SLNB guidelines and perceived utility of SLNB (Table 4). There was also considerable variation in the time point at which a GP would refer a patient, with some referring for biopsy, some performing biopsies but referring once a melanoma diagnosis was confirmed, and others performing wide local excisions.

\section{Discussion}

The data indicate that while GPs have generally favourable attitudes to SLNB, and they understand that Breslow thickness is important for guiding selection of patients for SLNB, they lack knowledge about the Breslow thickness cut-off points specified in the guidelines for referral of patients eligible for SLNB. GPs who saw higher volumes of patients with invasive melanoma were more likely to be familiar with the melanoma management guidelines; however, they were in the minority, and many of the participants did not feel sufficiently informed about the role of SLNB or which investigations should be performed at the time of SLNB. GPs reporting exposure to articles or talks about SLNB in the past three years were more likely to believe SLNB had an important role and to discuss SLNB with their patients.

For GPs who would discuss SLNB with and recommend SLNB to an eligible patient with a newly diagnosed melanoma, Breslow thickness was the most common reason for recommending SLNB, followed by lymphovascular invasion. Interestingly, the presence of palpable lymph nodes was also a common reason for recommending SLNB, even though a palpable node implies that nodal metastases are already present, negating the need for SLNB. Familiarity with recommendations for SLNB according to Breslow thickness varied among GPs. Of GPs who indicated that they would consider discussing
Table 2. General practitioner's recommendation for sentinel lymph node biopsy

Question relating to recommending sentinel

lymph node biopsy (SLNB)

Would you usually discuss and recommend biopsy to a patient with a newly diagnosed melanoma, if they were eligible for SLNB?*

\begin{tabular}{lr}
\hline No & 64 (28) \\
\hline Yes & 164 (72) \\
\hline Do you think that SLNB has an important role in the management of patients with melanoma?* \\
\hline No & 5 (2) \\
\hline Yes & 154 (68) \\
\hline Unsure & 69 (30) \\
\hline Have you read articles or listened to talks about SLNB in the past three years? & 92 (40) \\
\hline No & 137 (60) \\
\hline Yes & (3) \\
\hline${ }^{*}$ Data missing from three respondents & (6) \\
\hline
\end{tabular}

SLNB with or recommending SLNB for patients with melanoma, the most frequently selected Breslow thickness cut-off points were $>1.0-2.0 \mathrm{~mm}$ (37\% of GPs) and > $2 \mathrm{~mm}$ ( $30 \%$ of GPs). The guidelines recommend that SLNB be considered for all patients with a Breslow thickness of $>1.0 \mathrm{~mm}$ and for patients with Breslow thickness 0.8-1.0 mm if they have other high-risk pathological features. Patients with intermediate-thickness melanomas may have better diseasefree survival and lower rates of local and regional recurrence following SLNB. ${ }^{17}$ In addition, SLNB has been shown to have prognostic value for patients with thick ( $>4 \mathrm{~mm}$ ) clinically lymph node-negative melanoma. ${ }^{18}$

The results of this study indicate that many GPs do not perceive the SLNB guidelines as being relevant to them and do not know enough about SLNB to recommend the procedure. The study did not assess the details of how SLNB is discussed or communicated with patients, how that might differ with level of knowledge or how many patients may have declined SLNB; this is an area that could be addressed in future studies. The present findings are based on self-report and may be subject to social desirability bias; for example, if some GPs inaccurately reported being familiar with the guidelines for melanoma management. GPs may claim they 'know' the guidelines from information gained from their peers and opinion leaders but may not necessarily have a good understanding of them. ${ }^{19}$ Lack of knowledge of the guidelines may not be problematic (refer to 'Key points' in Table 4) if GPs are not performing biopsies of suspicious lesions; however, for GPs who do perform biopsies of suspicious lesions, it is likely some melanomas will be $>0.08 \mathrm{~mm}$ in thickness. In this case, it is important that the GPs appreciate how the guidelines relating to SLNB may affect their practice and their referral decisions, and therefore a patient's access to contemporary melanoma treatment including adjuvant therapy post-surgical resection. This barrier could be addressed through GP education. Another possibility for improving adherence to guidelines so that all eligible patients can be offered 
Table 3. General practitioners' knowledge of guidelines relating to sentinel lymph node biopsy and management of patients with an invasive melanoma $(n=164)^{*}$

Question

Which Breslow depth would you use as a criterion for recommending SLNB? ${ }^{\dagger}$

$<0.8 \mathrm{~mm}$

$<0.8 \mathrm{~mm}$ and other high-risk pathological feature/s

37 (23)

$0.8-1.0 \mathrm{~mm}$ $30(19)$

0.8-1.0 mm and other high-risk pathological feature/s

$101(63)$

$>1.0-2.0 \mathrm{~mm}$

62 (39)

$>2.0-4.0 \mathrm{~mm}$

$49(30)$

$>4.0 \mathrm{~mm}$

$48(30)$

Other ${ }^{\ddagger}$

$11(7)$

Which factors would influence your decision to discuss or recommend SLNB?

Breslow thickness

$151(92)$

Lymphovascular invasion $108(66)$

Presence of palpable lymph nodes

$110(67)$

The likelihood that the result will influence patient management

Body site of the melanoma

$88(54)$

Histopathological subtype

$82(50)$

Mitotic rate of melanoma

$82(50)$

Mitotic rate of melanoma

$80(49)$

Presence of ulceration 74 (45)

Comorbidities of the patient

$73(45)$

Morbidity of the SLN procedure

62 (38)

Access to services for SLN procedure mapping and biopsy

$59(36)$

Patient preference

$60(37)$

Age of the patient $70(43)$

The morbidity of completion lymphadenectomy $44(27)$

Distance to services for SLN procedure mapping and biopsy 45 (27)

Costs to the patient $43(26)$

Patient level of anxiety

44 (27)

Other

Missing data

\section{Reasons that SLNB may be of value}

More accurate staging and prognostic value

To assess suitability for adjuvant systemic therapies for patients who are SLNB positive

Influence of results on patient management

$106(67)$

Likely survival benefit $98(62)$

To select patients for completion lymphadenectomy $63(40)$

$48(30)$
Question

n (\%)

To whom would you refer a patient for definitive management if SLNB would be suitable?

A specialist melanoma service with a multidisciplinary team 49 (30)

A melanoma-trained surgical oncologist 35 (21)

\begin{tabular}{ll}
\hline A local general surgeon & 22 (13)
\end{tabular}

A melanoma-trained plastic surgeon 17 (10)

A melanoma specialist dermatologist 8 (5)

\begin{tabular}{ll}
\hline Any surgical oncologist & 6 (4)
\end{tabular}

Any plastic surgeon 5 (3)

Any dermatologist 6 (4)

A skin cancer clinic colleague 2 (1)

Other, including multiples of the above ${ }^{\S} 14$ (9)

Would you expect the clinician to whom you refer the patient to recommend SLNB?

Occasionally 23 (13)

Most of the time 70 (43)

\begin{tabular}{ll}
\hline Always & $71(43)$
\end{tabular}

What tests would you arrange pre-procedure for patients eligible for SLNB? ${ }^{+}$

No other tests or scans 45 (28)

CT of the abdomen, chest and pelvis 59 (36)

Whole-body PET/CT 46 (28)

Ultrasonography examination of all regional nodes 29 (18)

Chest X-ray 18 (11)

CT or MRI of the brain 15 (9)

After a positive SLNB, are you wanting to be involved in ongoing patient follow-up?

No

$7(4)$

Yes, with care managed by myself 4 (2)

Yes, with care managed by the specialist 62 (38)

Yes, with care managed in a shared care arrangement 91 (56)

After a negative SLNB, are you wanting to be involved in ongoing patient follow-up?

No

Yes, with care managed by myself

$2(1)$

Yes, with care managed by the specialist 42 (26)

Yes, with care managed in a shared care arrangement 92 (57)

*This table is based on the subset of GPs who said that they would usually discuss SLNB with and recommend SLNB to their patients, if they were eligible for SLNB.

${ }^{t}$ Respondents could select more than one answer.

₹Refer on to a surgeon who would decide on management or the GP would check the guideline when required

${ }^{\S}$ GPS who noted multiple options

$C T$, computed tomography; GP, general practitioner; MRI, magnetic resonance imaging; PET, positron emission tomography; SLN, sentinel lymph node; SLNB,

sentinel lymph node biopsy 
Table 4. Interview data on general practitioners' experiences and attitudes to sentinel lymph node biopsy for patients with melanoma

\begin{tabular}{|c|c|c|c|}
\hline Factors & Range of responses & Illustrative quotes & Key points in relation to GP practice \\
\hline \multirow[t]{2}{*}{$\begin{array}{l}\text { Perceived utility of } \\
\text { SLNB }\end{array}$} & $\begin{array}{l}\text { Only useful for staging/ } \\
\text { prognostication }\end{array}$ & $\begin{array}{l}\text { 'I think it's good prognostically, but as far as everything } \\
\text { else, I'm not sure of the utility [of SLNB].' (ID 97; } \\
\text { independent GP) }\end{array}$ & \multirow{2}{*}{$\begin{array}{l}\text { Although many GPs indicated that } \\
\text { SLNB might be useful to guide } \\
\text { management decisions, and that } \\
\text { systemic adjuvant therapies were } \\
\text { important in melanoma treatment, } \\
\text { not all GPs seemed to be explicitly } \\
\text { aware that SLNB is the procedure } \\
\text { that is currently being used to } \\
\text { identify patients who might benefit } \\
\text { from systemic adjuvant therapies. }\end{array}$} \\
\hline & $\begin{array}{l}\text { Useful for staging/ } \\
\text { prognostication and } \\
\text { to guide treatment } \\
\text { management, including } \\
\text { access to adjuvant } \\
\text { therapies }\end{array}$ & $\begin{array}{l}\text { 'Well I think [access to systemic adjuvant therapies] } \\
\text { is really probably one of the biggest reasons why you } \\
\text { would do a sentinel node biopsy in this day and age. } \\
\text { It was a little different early on before we had any } \\
\text { big systemic therapies because if you had a positive } \\
\text { sentinel node, you really had a worse prognosis, but } \\
\text { then nothing you could really do about it, wouldn't }\end{array}$ & \\
\hline
\end{tabular}

then nothing you could really do about it, wouldn't make any real difference except to give you that information and for a lot of patients that was enough, that they just wanted to know a better idea of what their prognosis might be. But now that you've actually got options for adjuvant therapy or neoadjuvant therapy, I think that's a really big plus in favour of actually having that sentinel node biopsy done and seeing someone who's in that melanoma field who is aware of exactly what might often be offered if that came back as positive.' (ID 233; skin cancer clinic GP)

\begin{tabular}{lll}
\hline $\begin{array}{l}\text { Perceived relevance } \\
\text { of guidelines relating } \\
\text { to SLNB }\end{array}$ & $\begin{array}{l}\text { Not relevant to GPs' } \\
\text { practice }\end{array}$ & $\begin{array}{l}\text { '[The SLNB guidelines] don't affect us, so I don't think } \\
\text { I've seen any.' (ID 120; medical centre GP) }\end{array}$ \\
\cline { 2 - 3 } & $\begin{array}{l}\text { Relevant to GPs' } \\
\text { practice }\end{array}$ & $\begin{array}{l}\text { 'Oh yeah, definitely. I've read [the new SLNB } \\
\text { guidelines]. I've gone through it and I have read through } \\
\text { it and we've had a few forums where we've discussed } \\
\text { the new guidelines regarding it.' (ID 208; skin cancer } \\
\text { clinic GP) }\end{array}$ \\
\end{tabular}

Working knowledge of guidelines relating to SLNB
Limited or no knowledge of guidelines relating to SLNB

Explicit knowledge of guidelines relating to SLNB
'Oh, so [knowledge of guidelines for SLNB] is getting a little bit past what I do.' (ID 10; medical centre GP)

'Well you get the histology back and it says melanoma, then I refer them to the surgeon to have a wider excision. Yeah, then depending on what the level of the melanoma is, how thick it is, they do the sentinel node biopsy.' (ID 92; medical centre GP)

'So, any patient, anything greater than $0.8 \mathrm{~mm}$ I talk to them about sentinel lymph node biopsies and whether it would be beneficial for them or not. Then upon discussion, often they will get referred on to the melanoma unit to get further discussion and whether or not they want to go ahead with it.' (ID 208; skin cancer clinic GP)

\begin{tabular}{ll}
\hline $\begin{array}{l}\text { Appreciation of how } \\
\text { SLNB relates to care }\end{array}$ & $\begin{array}{l}\text { Limited appreciation } \\
\text { of when and for whom }\end{array}$ \\
of a patient with & SLNB is indicated \\
melanoma &
\end{tabular}
melanoma
'[My understanding of the role of SLNB in melanoma] is very cursory, like I know that it's an indication then of spread, but yeah, I guess I'm not as clear about when we need to do [an SLNB] and in which patients.' (ID 5; independent GP)

'I don't know exactly the Breslow thickness [for an SLNB] off by heart. I know it's important.' (ID 135; GP in government clinic]

Explicit appreciation of when and for whom SLNB is indicated
'Well I think [SLNB] is quite important and, like I said, if I think that they need sentinel node biopsy, I don't do the re-excision, I send them to the specialist and then go from there.' (ID 7; medical centre GP)

'My advice is anything with a Breslow thickness of greater than one millimetre, or greater than 0.75 in height and is high risk pathology, is a sentinel lymph node biopsy.' (ID 214; skin cancer clinic GP)
Participants were not homogeneous in their knowledge of, or attitudes towards, SLNB and the guidelines relating to SLNB.

- The only GPs for whom SLNB guidelines are not at all relevant are those GPs who do not have any involvement in skin cancer: that is, the GP would immediately refer on for biopsy any patient who presents with a suspicious lesion that could potentially be a melanoma.

- For all other GPs - that is, GPs who biopsy suspicious lesions or perform wide local excision on confirmed melanomas - it is important that the GP appreciates how the guidelines relating to SLNB may affect their practice and their referral decisions.
Lack of detailed knowledge of guidelines relating to SLNB may affect management:

- If a GP is referring all patients for biopsy or wide local excision (for confirmed melanomas), then it becomes the specialist's responsibility to discuss SLNB with the patient.

- Lack of detailed knowledge of SLNB guidelines (and therefore the indications for referral for SLNB) is problematic if a GP performs a wide local excision without considering suitability for SLNB (which should be performed at the same time as wide local excision). 
the procedure is the inclusion of a comment in the histopathological report of a melanoma along the lines of: 'SLNB should be a consideration for this patient. If it is to be performed, it should be done at the same time as wide local excision'.

There is also evidence that inappropriate tests are being ordered for patients following diagnosis of melanoma. The guidelines indicate there is no evidence to support chest X-rays, PET/ CT or magnetic resonance imaging for initial staging of patients with thin or intermediate Breslow thickness primary melanoma (Stage I-IIB). ${ }^{20} \mathrm{PET} / \mathrm{CT}$ for Stage IIC patients may be considered. Nodal ultrasonography is recommended only when SLNB has been declined or is not possible, ${ }^{21}$ since ultrasonography has lower sensitivity and specificity when compared with SLNB. ${ }^{22}$ Access to facilities was noted as a barrier to performing SLNB by some GPs based in regional and remote centres, and not all eligible patients are referred to clinicians who perform SLNB.

A slight majority (56\%) of GPs preferred to manage patient follow-up in a shared care arrangement, which relies on good communication between the GP and specialist and clear delineation of roles. ${ }^{23,24}$ A limitation of the present study is that it was not possible to compare the results for GPs working in skin cancer clinics against those working in general practice because of relatively few GPs from skin cancer clinics participating in the study. A third of GPs were unsure about the role of SLNB, which might be related to general lack of awareness or to conflicting information in journals and presentations on the benefits and harms of SLNB. ${ }^{9-14} \mathrm{~A}$ strength of the study is that a relatively large and representative sample of GPs was surveyed. These findings are most relevant to Australia but may be applicable to other countries with comparable health systems and melanoma management practices. Difficulties with and resistance to adhering to guideline recommendations have been acknowledged by GPs in other settings. ${ }^{25-27}$ Reasons for non-compliance to guidelines by GPs included limited knowledge, which was affected by the time available to search for information while consulting, and the perception that guidelines may not take into account specific patient needs. Resistance may also be experienced as a result of beliefs that guidelines are 'imposed' or not trusting recommendations. ${ }^{23-25}$

Some people argue that SLNB is never indicated because of the lack of survival benefit; however, all evidencebased guidelines - including national (Australian, The National Comprehensive Cancer Network, British Association of Dermatologists) and international (European Society for Medical Oncology) guidelines - recommend that SLNB be considered for a melanoma $>1.0 \mathrm{~mm}$ in thickness or $>0.8 \mathrm{~mm}$ Breslow thickness with additional high-risk features.

\section{Conclusions}

GPs are at the front line of melanoma management in Australia. Although GPs had a generally satisfactory level of understanding of the benefits of SLNB, many were not familiar with the guidelines regarding which patients should be considered for SLNB, and there was evidence that inappropriate investigative tests may be ordered prior to referral. These results indicate a need for further education and discussion with GPs about the role of SLNB in melanoma management, targeting those who biopsy or excise melanomas. For GPs who are not familiar with the melanoma guidelines or who are not confident in managing primary melanoma, all patients with invasive melanoma should ideally be referred to specialised units for management. These findings will inform the development, dissemination and evaluation of education strategies and other initiatives to improve GPs' knowledge of SLNB and ultimately lead to better patient outcomes.

\section{Authors}

Caroline G Watts MPH, PhD, Research Fellow, Cancer Epidemiology and Prevention Research, Sydney School of Public Health, The University of Sydney, NSW; Research Fellow, Surveillance, Evaluation and Research Program, Kirby Institute, UNSW, NSW. caroline.watts@sydney.edu.au

Andrea L Smith MPH, PhD, Research Fellow, Centre for Healthcare Resilience and Implementation Science (CHRIS), Australian Institute of Health Innovation (AIHI), Faculty of Medicine and Health Sciences, Macquarie University, NSW
Sam Robinson BAppPH, Research Officer, Cancer Epidemiology and Prevention Research, Sydney School of Public Health, University of Sydney, NSW Chiao-Han (Sharon) Chang MD, Research Assistant, Cancer Epidemiology and Prevention Research, Sydney School of Public Health, University of Sydney, NSW Chris Goumas MPH, Biostatistician, Cancer Epidemiology and Prevention Research, Sydney School of Public Health, University of Sydney, NSW Helen Schmid MPH, Co-ordinator, Melanoma Research Program, Westmead institute for Medical Research, NSW

John W Kelly MD, FACD, Consultant Dermatologist, Victorian Melanoma Service, The Alfred, Vic;

Adjunct Professor, Monash University, Department of Medicine at Alfred Health, Vic

Angela M Hong MBBS, MMed, PhD, FRANZCR, Radiation Oncologist, Melanoma Institute Australia, Chris O'Brien Lifehouse, Mater Hospital North Sydney, NSW; Clinical Professor, Medicine, Central Clinical School, University of Sydney, NSW

Richard A Scolyer BMedSci, MBBS, MD, FRCPA, FRCPath, Clinical Professor, Pathology, Central Clinical School, University of Sydney, NSW: Co-Medical Director and Consultant Pathologist, Melanoma Institute Australia, NSW; Senior Staff Specialist, Tissue Pathology and Diagnostic Oncology, Royal Prince Alfred Hospital, NSW Georgina V Long BSc, PhD, MBBS, FRACP, CoMedical Director, Melanoma Institute Australia, Vic; Chair of Melanoma Medical Oncology and Translational Research, Melanoma Institute Australia and Royal North Shore Hospital, University of Sydney, NSW

Andrew J Spillane MD, FRACS, MBBS, BMedSc, CCPU, Professor of Surgical Oncology, University of Sydney, Northern Clinical School, NSW

Michael Henderson MBBS, BMedSc, MD, FRACS, Professor of Surgery, University of Melbourne, Vic; Surgeon, Melanoma and Skin Service, Breast Service and Sarcoma Service, Peter MacCallum Cancer Centre, Vic

David E Gyorki MBBS, MD, FRACS, Consultant surgeon, Peter MacCallum Cancer Centre, Vic

Victoria J Mar MBBS (Hons), FACD, PhD, Director of the Victorian Melanoma Service, The Alfred, Vic; Adjunct Associate Professor, School of Public Health and Preventive Medicine, Monash University Vic Rachael L Morton MScMed, PhD (Clin Epi) (Hons), Director of Health Economics, NHMRC Clinical Trials Centre, Sydney Medical School, University of Sydney, NSW; Professor of Health Economics, Sydney Medical School, University of Sydney, NSW Robyn PM Saw FRACS, MS, MBBS, Associate Professor in Surgery, Central Clinical School, University of Sydney, NSW; Surgical Oncologist, Melanoma Institute Australia, NSW; General Surgeon, Royal Prince Alfred Hospital, NSW Alex H Varey BSc (Med), MBBS (Hons), FRACP, PhD, Medical Oncologist, Melanoma Institute Australia Royal North Shore and Mater Hospitals, NSW; Associate Professor, Melanoma Medical Oncology, University of Sydney, NSW

Graham J Mann MBBS, PhD, FRACP, Director, John Curtin School of Medical Research, Australian National University, ACT; Principal Investigator, Melanoma Institute Australia, University of Sydney, NSW

John F Thompson AO, MD, FRACS, FACS, Professor of Melanoma and Surgical Oncology, University of Sydney, NSW

Anne E Cust MPH (Hons), PhD, Head of Cancer Epidemiology and Prevention Research Group, Sydney School of Public Health and Melanoma Institute Australia, University of Sydney, NSW; 
Professor of Cancer Epidemiology, Sydney Medical School, University of Sydney, NSW

Competing interests: GVL is a Consultant Advisor and reports personal fees from Aduro, Amgen, Array, BMS, Idera, Incyte, MSD, Novartis, Oncosec, Pierre Fabre, Roche and Sandoz, outside the submitted work. DEG is a member of the Amgen advisory board. RPMS reports personal fees from Novartis, MSD and BMS, outside the submitted work. JFT reports personal fees, travel support and honoraria for advisory board participation from GSK and Provectus Inc, and personal fees from MSD and BMS, outside the submitted work. RAS reports grants from the NHMRC (Program Grant and Practitioner Fellowship Grant), non-financial support from Melanoma Institute Australia and The Ainsworth Foundation, salary from Royal Prince Alfred Hospital, and fees for professional services from MSD, GSK, BMS, Dermpedia, Novartis, Myriad, NeraCare GmbH and Amgen, outside the submitted work. VJM reports personal fees from BMS and MSD, outside the submitted work.

Funding: CW is supported by the NHMRC Centre of Research Excellence in Melanoma (1135285) and The University of Sydney Kickstart grant. AEC is supported by an NHMRC Program grant (1093017), an NHMRC Career Development Fellowship (1147843), a Cancer Institute NSW Fellowship (15/CDF/1-14) and The University of Sydney SOAR Fellowship. RLM is supported by an NHMRC TRIP Fellowship (1150989). RAS is supported by an NHMRC Practitioner Fellowship.

Provenance and peer review: Not commissioned, externally peer reviewed.

\section{Acknowledgements}

The authors would like to thank all GPs who participated in the study and Ms Katrina Tune from HealthCert for facilitating their attendance at the cancer skills workshop. Support from colleagues at the Melanoma Institute Australia is also gratefully acknowledged.

\section{References}

1. Wilkinson D, Askew DA, Dixon A. Skin cancer clinics in Australia: Workload profile and performance indicators from an analysis of billing data. Med J Aust 2006;184(4):162-64.

2. Watts CG, Madronio CM, Morton RL, et al. Diagnosis and clinical management of melanoma patients at higher risk of a new primary melanoma: A population-based study in New South Wales, Australia. Australas J Dermatol 2017;58(4):278-85. doi: 10.1111/ajd.12530.

3. Morton DL, Wen DR, Wong JH, et al. Technical details of intraoperative lymphatic mapping for early stage melanoma. Arch Surg 1992;127(4):39299. doi: 10.1001/archsurg.1992.01420040034005.

4. Gershenwald JE, Scolyer RA, Hess KR, et al. Melanoma staging: Evidence-based changes in the American Joint Committee on Cancer eighth edition cancer staging manual. CA Cancer J Clin 2017;67(6):472-92. doi: 10.3322/caac.21409.

5. Gyorki D, Barbour A, Mar V, Sandhu S, Hanikeri M. Cancer Council Australia Melanoma Guidelines Working Party. When is a sentinel node biopsy indicated? Sydney: Cancer Council Australia, 2018. Available at https://wiki.cancer.org.au/ australia/Clinical_question:When_is_a_sentinel_ node_biopsy_indicated\%3F [Accessed 3 February 2020].

6. Varey AHR, Madronio CM, Cust AE, et al. Poor adherence to national clinical management guidelines: A population-based, cross-sectional study of the surgical management of melanoma in New South Wales, Australia. Ann Surg Oncol 2017;24(8):2080-88. doi: 10.1245/s10434-0175890-7.

7. Morton DL, Thompson JF, Cochran AJ, et al. Final trial report of sentinel-node biopsy versus nodal observation in melanoma. N Engl J Med 2014;370(7):599-609. doi: 10.1056/ NEJMoa1310460.

8. Bigby M, Zagarella S, Sladden M, Popescu CM. Time to reconsider the role of sentinel lymph node biopsy in melanoma. J Am Acad Dermatol 2019;80(4):1168-71. doi: 10.1016/j.jaad.2018.11.026.

9. Faries MB, Cochran AJ, Thompson JF. Re: 'Time to reconsider the role of sentinel lymph node biopsy in melanoma'. J Am Acad Dermatol 2019;pii:S0190-9622(19)30508-0. doi: 10.1016/j. jaad.2019.03.064

10. Sladden M, Zagarella S, Popescu C, Bigby M. No survival benefit for patients with melanoma undergoing sentinel lymph node biopsy: Critical appraisal of the Multicenter Selective Lymphadenectomy Trial-I final report. Br J Dermatol 2015:172(3):566-71. doi: 10.1111/ bjd.13675.

11. Faries MB, Cochran AJ, Elashoff RM, Thompson JF. Multicenter Selective Lymphadenectomy Trial-I confirms the central role of sentinel node biopsy in contemporary melanoma management: Response to 'No survival benefit for patients with melanoma undergoing sentinel lymph node biopsy: Critical appraisal of the Multicenter Selective Lymphadenectomy Trial-I final report'. Br J Dermatol 2015;172(3):57173. doi: 10.1111/bjd.13676.

12. Harris PA, Taylor R, Thielke R, Payne J, Gonzalez N, Conde JG. Research electronic data capture (REDCap) - A metadata-driven methodology and workflow process for providing translational research informatics support. J Biomed Inform 2009;42(2):377-81. doi: 10.1016/j.jbi.2008.08.010.

13. Australian Bureau of Statistics. The Australian Statistical Geography Standard (ASGS) remoteness structure. Canberra: ABS, 2018. Available at www.abs.gov.au/websitedbs/ D3310114.nsf/home/remoteness+structure [Accessed 3 Febuary 2020].

14. Australian Bureau of Statistics. Socio-Economic Indexes for Areas: SEIFA provides measures of socio-economic conditions by geographic area. Canberra: ABS, 2018. Available at www.abs.gov. $\mathrm{au} /$ websitedbs/censushome.nsf/home/seifa [Accessed 3 Febrary 2020].

15. von Elm E, Altman DG, Egger M, Pocock SJ, Gøtzsche PC, Vandenbroucke JP. The Strengthening the Reporting of Observational Studies in Epidemiology (STROBE) statement: Guidelines for reporting observational studies. Int J Surg 2014;12(12):1495-99. doi: 10.1016/j. ijsu.2014.07.013.

16. Braun V, Clarke V. Using thematic analysis in psychology. Qual Res Psychol 2006;3(2):77-101. doi: 10.1191/1478088706qp063oa.

17. Kyrgidis A, Tzellos T, Mocellin S, et al. Sentine lymph node biopsy followed by lymph node dissection for localised primary cutaneous melanoma. Cochrane Database Syst Rev 2015(5):CD010307. doi: 10.1002/14651858. CD010307.pub2.

18. Ribero S, Osella-Abate S, Sanlorenzo M, et al. Sentinel lymph node biopsy in thick-melanoma patients $(\mathrm{N}=350)$ : What is its prognostic role? Ann Surg Oncol 2015;22(6):1967-73. doi: 10.1245/ s10434-014-4211-7.
19. Gabbay J, le May A. Evidence based guidelines or collectively constructed 'mindlines?' Ethnographic study of knowledge management in primary care. BMJ 2004;329(7473):1013. doi: 10.1136/ bmj.329.7473.1013.

20. Cancer Council Australia Melanoma Guidelines Working Party. Summary of reccomendations. Sydney: Cancer Council Australia, 2019. Available at https://wiki.cancer.org.au/ australia/Guidelines:Melanoma/Summary of recommendations [Accessed 3 February 2020].

21. Bafounta ML, Beauchet A, Chagnon S, Saiag P. Ultrasonography or palpation for detection of melanoma nodal invasion: A meta-analysis. Lancet Oncol 2004;5(11):673-80. doi: 10.1016/s14702045(04)01609-2.

22. Ipenburg NA, Thompson JF, Uren RF, Chung D, Nieweg OE. Focused ultrasound surveillance of lymph nodes following lymphoscintigraphy without sentinel node biopsy: A useful and safe strategy in elderly or frail melanoma patients. Ann Surg Oncol 2019;26(9):2855-63. doi: 10.1245/ s10434-019-07505-6.

23. Turbitt E, Kunin M, Gafforini S, Sanci L, Spike N, Freed GL. Perspectives of Australian general practitioners on shared care for paediatric patients. Aust J Prim Health 2017;23(2):147-53. doi: $10.1071 /$ py16002.

24. Foy R, Hempel S, Rubenstein L, et al. Metaanalysis: Effect of interactive communication between collaborating primary care physicians and specialists. Ann Intern Med 2010;152(4):247-58. doi: 10.7326/0003-4819-152-4-201002160-00010.

25. Egerton T, Diamond LE, Buchbinder $\mathrm{R}$, et al. A systematic review and evidence synthesis of qualitative studies to identify primary care clinicians' barriers and enablers to the management of osteoarthritis. Osteoarthritis Cartilage 2017;25(5):625-38. doi: 10.1016/j. joca.2016.12.002.

26. Basedow M, Runciman WB, Lipworth W, et al. Australian general practitioner attitudes to clinical practice guidelines and some implications for translating osteoarthritis care into practice. Aust J Prim Health 2016;22(5):403-08. doi: 10.1071/ py15079.

27. Slade SC, Kent P, Patel S, et al. Barriers to primary care clinician adherence to clinical guidelines for the management of low back pain: A systematic review and metasynthesis of qualitative studies. Clin J Pain 2016:32(9):800-16. doi: 10.1097/ ajp.0000000000000324. 